\title{
Nanoplasmonic hydrogen sensing
}

\author{
Carl Wadell, Svetlana Syrenova and Christoph Langhammer \\ Department of Applied Physics, Chalmers University of Technology, 41296 Göteborg, Sweden
}

\begin{abstract}
In this review we discuss the evolution of surface plasmon resonance and localized surface plasmon resonance based hydrogen sensors. We put particular focus on how they are used to study metal-hydrogen interactions at the nanoscale, both at the ensemble and the single nanoparticle level. Such efforts are motivated by a fundamental interest in understanding the role of nanosizing on metal hydride formation processes. However, nanoplasmonic hydrogen sensors are not only of academic interest but may also find more practical use as all-optical gas detectors in industrial and medical applications, as well in a future hydrogen economy, where hydrogen is used as a carbon free energy carrier.
\end{abstract}

\section{INTRODUCTION}

\subsection{Why do we need hydrogen sensors?}

Hydrogen is envisioned to be a clean and renewable energy carrier for the fuel of the future. The challenge stands in optimizing costs, reliability and safety at all stages of hydrogen production, distribution, storage and utilization as a fuel. ${ }^{1}$ Thus, from materials science perspective hydrogen sensing is required for understanding and solving these issues. However, hydrogen sensors are not only useful during the development of this technology. If hydrogen is introduced as the major energy carrier, hydrogen sensors will become a vital part of the infrastructure, ensuring safe operation. As of now hydrogen gas is largely involved in various areas of industry either being one of the necessary components or by/end products of the process. Currently, hydrogen is mainly used in two non-fuel applications such as production of ammonia for the fertilizer industry and refinement of crude oils in the petrochemical industry. Hydrogen sensors are needed in order to monitor and control hydrogen partial pressure for as safe and as efficient as possible processing. ${ }^{2}$ Besides industrial applications, hydrogen sensing can be useful when hydrogen is an end or by-product of a biological process. For example, in food industry detection of hydrogen produced by certain bacteria can help determine if the food is spoiled. ${ }^{3}$ Hydrogen sensing was also suggested to be used for indication of whether the food has been irradiated ${ }^{4}$, or for detection of leaks in flexible food packages by employing hydrogen as a tracer gas ${ }^{5}$. Also in medical applications an interest in hydrogen sensors has arisen, since hydrogen breath measurements have proven to be useful in the diagnostics of certain conditions ${ }^{6}$ such as lactose intolerance or bacterial overgrowth in intestines.

\subsection{What are the requirements for hydrogen sensors?}

Hydrogen sensors are imposed by a number of requirements that can be quite specific depending on application areas. These requirements can be generally summarized ${ }^{2,7,8}$ as: acceptable measurement range (e.g. $0.01-10 \%$ for leak detection), sufficient accuracy $( \pm 25$ or $50 \%)$ and reliability, quick response $(<30 \mathrm{~s})$ and recovery $(<60 \mathrm{~s})$ time, stable signal with good signal-to-noise ratio, robustness under environmental conditions (e.g. temperature $\left(-20\right.$ to $\left.50^{\circ} \mathrm{C}\right)$, pressure (80-110 kPa), relative humidity (10-98\%), gas flow rate). At the same time an ideal hydrogen sensor has to be explosion proof, have long lifetime and low cost, simple operation and maintenance, as well as simple system integration and interface. Other important characteristics of hydrogen sensors are low cross-sensitivity (e.g. towards hydrocarbons, carbon monoxide, hydrogen sulfide, sulfur dioxide etc.) and low susceptibility to contaminations and poisoning.

\subsection{Why optical hydrogen sensors?}

Several mechanisms for hydrogen sensing have been studied for more than a century. Traditional approaches include gas chromatographs, mass spectrometry, thermal conductivity sensors and laser gas analysis. There are also commercially available sensors based on a solid-state approach to hydrogen sensing such as catalytic sensors, electrochemical sensors,

Plasmonics: Metallic Nanostructures and Their Optical Properties XII, edited

by Allan D. Boardman, Proc. of SPIE Vol. 9163, 916310 - (c) 2014 SPIE

CCC code: $0277-786 X / 14 / \$ 18 \cdot$ doi: $10.1117 / 12.2063399$

Proc. of SPIE Vol. $9163916310-1$ 
resistance-based sensors, work function based sensors, as well as sensors with mechanical, acoustical and optical approaches. Comprehensive reviews on existing and emerging hydrogen sensing technologies mentioned above can be found in recent work by Hübert et al. ${ }^{9}$. Ando ${ }^{10}$ also specifically summarizes detection of various gases (including hydrogen) by means of optochemical sensors. Our review is focused on the recent developments of hydrogen sensing based on the phenomena of the surface plasmon resonance (SPR) at a metal dielectric interface and localized SPR (LSPR) in metal nanoparticles.

Optical sensors have a number of advantages compared to the traditional electrical sensors. The main advantage is that they pose no risk to generate sparks, something that could be devastating when operating in explosive hydrogen atmospheres. They also have the benefit of not being affected by electromagnetic interference. At the same time, as with any other technique, there are certain disadvantages with optical sensors. The main ones being interference from ambient light and, since many of the optical sensors are still based on Pd (as will be discussed below), they are prone to mechanical stress and ageing due to frequent exposure to hydrogen. Moreover they are also susceptible to sulfur poisoning from the air.

\section{HYDROGEN IN METALS}

In order to build a hydrogen detector some kind of "active" material that will specifically react in some measurable way to the presence of hydrogen is needed. Numerous different materials that fulfill this criterion exist, e.g. various metal oxides and polymers. However, in this review we focus on the transformation of a metal into a hydride in the presence of hydrogen ${ }^{11}$ as our signal transducer since the plasmon resonance phenomenon occurs in metals. The ability of some metals to absorb hydrogen atoms into interstitial sites in their lattice has received a lot of research attention with different applications in mind. The main interest is perhaps in its application as hydrogen storage medium for vehicles, owing to the huge volume reduction of the hydrogen when stored in a metal hydride compared to, for instance, high pressure gas tanks ${ }^{12}$. Also stationary hydrogen storage at a larger scale as well as metal-hydride batteries are important application areas of metal hydrides ${ }^{13}$. Hydride formation in metal nanoparticles has also been a hot topic as the sorption behavior is expected to differ greatly in nanosized entities compared to their bulk counterparts. ${ }^{14}$

As mentioned, the metal hydride formation can also be utilized in hydrogen sensors. To gain some insight into how these sensors work we first take a short look at how the hydride formation in a metal takes place. The basic process is sketched in Figure 1. As the metal is exposed to low hydrogen gas pressures some hydrogen molecules will dissociate on the metal surface (we note, however, that on many metals this is an activated process that does not occur spontaneously at ambient conditions) and the hydrogen atoms will start to diffuse into the metal lattice. At this stage a solid solution of hydrogen in the metal lattice (called the $\alpha$-phase, see Figure 1a) is formed and since the amount of hydrogen is rather low hydrogen-hydrogen interaction inside the lattice are minor. Nevertheless, as the hydrogen is introduced into the metal it will induce a local strain on the metal lattice. As the hydrogen pressure around the metal is increased even further, the amount of hydrogen in the metal lattice will increase and eventually hydrogen-hydrogen interactions (resulting from the lattice strain as well as electronic interactions) become appreciable, and the formation of regions of the hydride ( $\beta$-phase) starts. At this stage the $\alpha$-phase and $\beta$-phase coexist at equilibrium and an incremental increase in the hydrogen pressure surrounding the metal will only result in the growth of the $\beta$-phase regions. Eventually, with continued increased external hydrogen pressure, the entire metal will be transformed into the $\beta$-phase, the hydride formation is complete and any further increase in hydrogen pressure will only result in minor changes in the hydrogen content in the metal.

If one - at constant temperature - maps the hydrogen content in the metal versus the applied hydrogen pressure a socalled isotherm is obtained, see Figure 1b. The isotherm will exhibit a clear "plateau" at the pressure where the $\alpha$ and $\beta$ phase coexist, i.e. at the hydride formation pressure. As the temperature is increased, the pressure at which the hydride formation takes place also increases. In this way, by measuring isotherms at several temperatures, the phase diagram of the metal-hydrogen system can be mapped out.

If one instead decreases the hydrogen pressure around the hydride, the unloading process will take place in a similar way, also exhibiting a plateau as the hydride is decomposed. However, the hydride decomposition will in most cases not occur at the same pressure as the formation, but rather at a lower pressure. This hysteresis is also the result of the lattice strain induced by the presence of hydrogen in the metal lattice. This strain creates an energy barrier that needs to be surmounted in order for the hydride to decompose. ${ }^{15}$ 
(a)

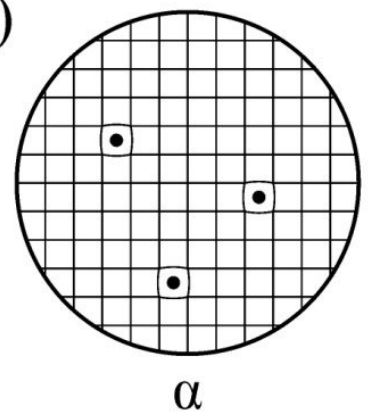

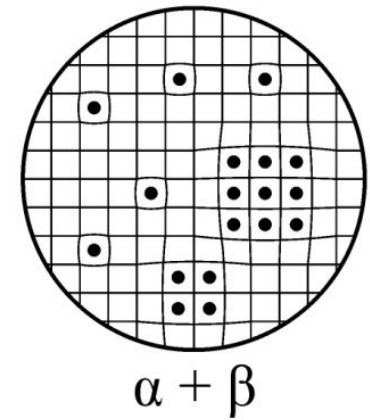

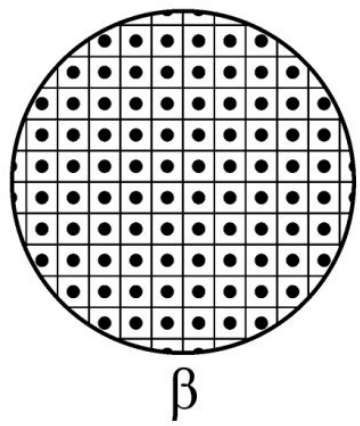

(b)

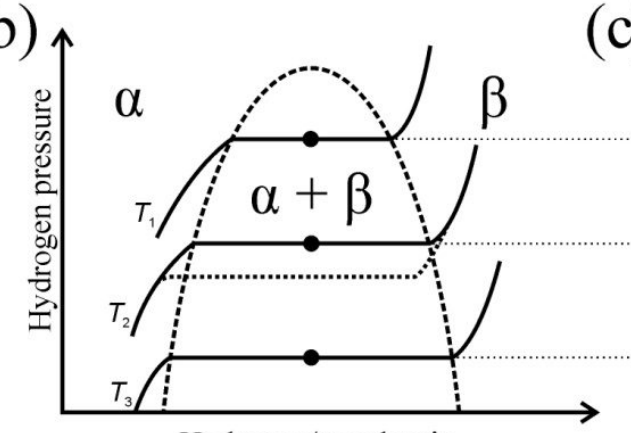

Hydrogen/metal ratio (c)

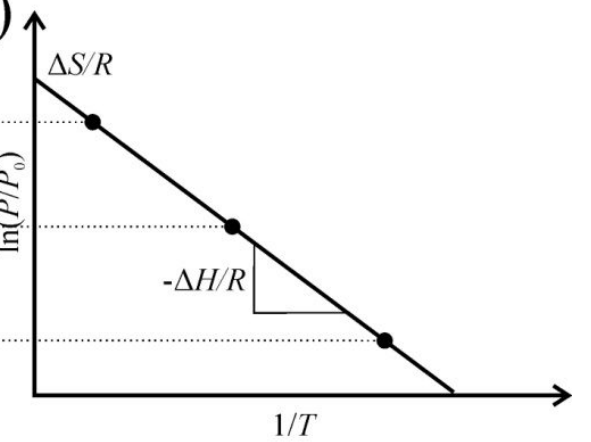

Figure 1. Sketch of the hydride formation process in a metal. a) The different stages during the hydride formation: $\alpha$-phase, mixed phase, and fully formed hydride ( $\beta$-phase). b) Sketch of isotherms showing a plateau at the hydride formation pressure for a given temperature. This plateau increases as the temperature increases. c) Van't Hoff analysis where change in enthalpy $(\Delta \mathrm{H})$ and entropy $(\Delta \mathrm{S})$ during the hydride formation are extracted from the temperature dependence of the plateau pressures.

From isotherm measurements carried out at different temperatures it is possible to extract thermodynamic data regarding the hydride formation/decomposition. At the plateau the chemical potentials of hydrogen in the gas phase and the hydride phase are equal by definition. This equality gives rise to the Van't Hoff equation:

$$
\ln \frac{P}{P_{0}}=-\frac{\Delta H}{R T}+\frac{\Delta S}{R}
$$

where $P$ is the plateau pressure, $P_{0}$ is atmospheric pressure, $\Delta H$ and $\Delta S$ are, respectively, the changes in enthalpy and entropy for the hydride formation/decomposition, $R$ is the gas constant, and $T$ is the temperature. By measuring isotherms at different temperatures and extracting the plateau pressures it is possible to use the Van't Hoff equation to extract $\Delta H$ and $\Delta S$. This is done using a Van't Hoff plot, see Figure 1c, in which $\ln \left(P / P_{0}\right)$ is plotted versus $1 / T$. From the obtained line $\Delta H$ and $\Delta S$ are determined by the slope of the line and its intersection with the y-axis, respectively.

\section{PLASMONIC SENSING}

As light impinges on a metal nanoparticle that is smaller or comparable to the wavelength of the light, resonant collective oscillations of the conduction electrons in the nanoparticle can be excited. This resonance is called a localized surface plasmon resonance (LSPR), see Figure 2a. At the wavelength where the resonance occurs, the metal nanoparticles will strongly absorb and scatter light. This phenomenon was first reported by Faraday ${ }^{16}$ in 1857 as he studied coloration of gold nanoparticle colloids. However, the principle had already been used for centuries to stain glass (e.g. church windows). Because of charge separation at the surface of the nanoparticle as the resonance is excited, a strongly enhanced electric field will be generated locally around the nanoparticle, see Figure $2 \mathrm{~b}$. The wavelength at which the LSPR occurs depends on several factors including the size, shape and material of the nanoparticle; but it also depends on 
the environment around it. Therefore, by tracking the LSPR wavelength it is possible to detect any changes to the nanoparticle itself, or to its immediate surroundings. This is the basis for plasmonic sensing. ${ }^{17}$

Similarly to the metal nanoparticle case it is also possible to excite resonant collective electron excitations on metal films at the interface towards a dielectric, see Figure 2c. On a film the resonance is no longer localized, as in the nanoparticles, but can be understood as a freely propagating surface charge density wave. These resonances are therefore simply called surface plasmon resonances (SPR). Because of the charge separation along the surface of the metal an evanescent electric field will be generated. SPRs can thus be used for sensing in a similar fashion as the LSPRs. However, unlike the LSPRs, SPRs cannot be excited directly by light at normal incidence. The light needs to be provided momentum to excite the SPR, which is most commonly achieved by the use of a prism coupler, a diffraction grating or a waveguide, see Figure $5 a^{18}$.
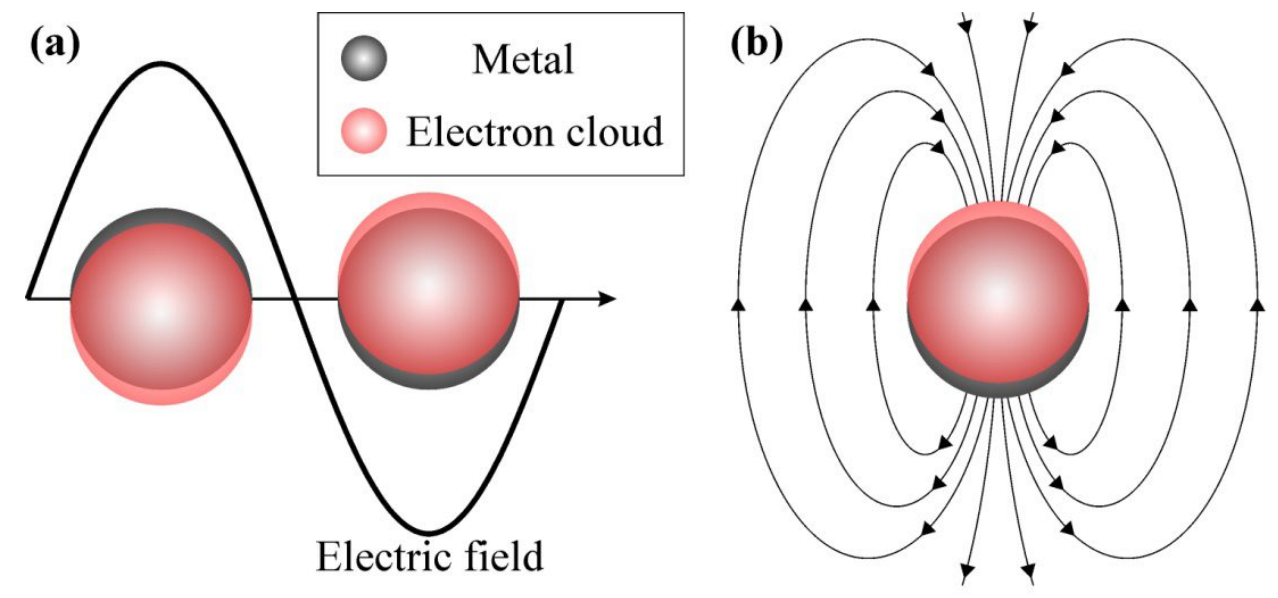

(c)

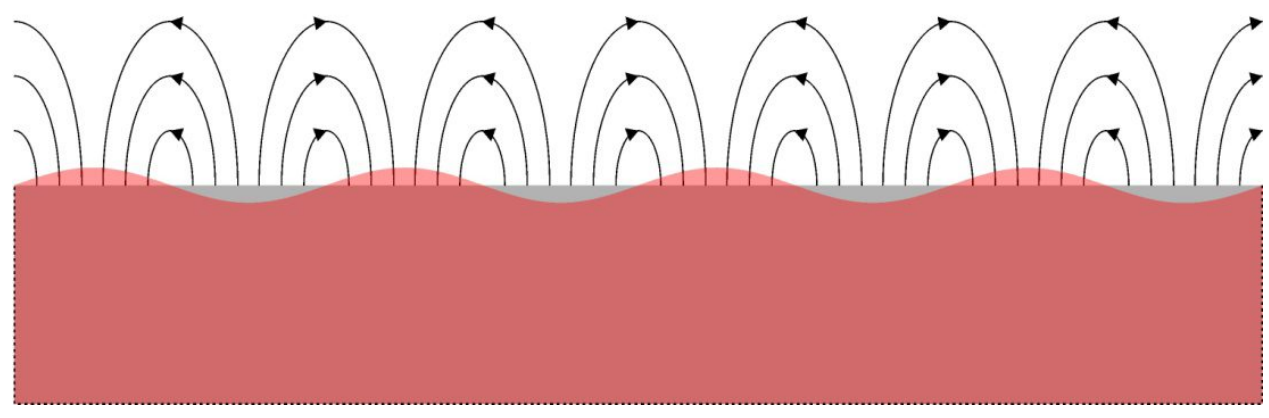

Figure 2. a) Sketch of the localized surface plasmon resonance (LSPR) in a metal nanoparticle as it is subjected to a time-dependent electric field, such as light. b) The charge separation of the surface of the metal nanoparticle caused by the LSPR generates a greatly enhanced electric field near the nanoparticle. c) Similar to the nanoparticle case, at the surface of a metal towards a dielectric, a surface plasmon resonance (SPR) can be excited under the right conditions. Also here the surface charge variations result in an evanescent electric field close to the surface of the metal film.

\section{LSPR-BASED HYDROGEN SENSORS}

\subsection{Direct sensors}

Direct LSPR hydrogen sensors consist of hydride forming metal nanoparticles, such as Pd. The LSPR of these nanoparticles themselves are utilized to monitor the hydrogen uptake in the metal. As hydrogen is absorbed into the metal lattice, the lattice will expand and also the dielectric function of the metal will change. ${ }^{19}$ These effects will both influence the resonance conditions for the LSP, which is used as the readout of the sensor. We call this direct sensing as 
the hydride forming entities themselves also provide the LSPR response used as the readout. An early example of such sensors is the work by Langhammer et al. ${ }^{20}$ where Pd nanodisks on a glass support are used as the sensing platform. In this work the sensor arrangement was inserted into a vacuum chamber and the hydrogen uptake/release was controlled by varying the pressure of hydrogen gas in the chamber. The chamber was equipped with windows making it possible to monitor the LSPR resonance of the sample by studying the transmission through the sample. In a later paper ${ }^{21}$ this work was extended by also studying the temperature dependence of the hydrogen uptake and release isotherms to extract thermodynamic information about the system according to the procedure described in the introduction, see Figure 3a. To verify that the LSPR shifts in these measurement indeed stem from the hydrogen uptake in the Pd nanoparticles, a quartz crystal microbalance (QCM) was used ${ }^{21,22}$ and Poyli et al. ${ }^{23}$ also theoretically verified the obtained experimental results based on first-principles calculations. Apart from Pd nanodisks, other nanostructures have been developed for direct LSPR hydrogen sensing, e.g. Pd nanorings ${ }^{24}$ and concave Pd nanocubes ${ }^{25}$. In the work by Strohfeldt et al. ${ }^{26}$ these sensors have been taken one step further, by not only considering a Pd disk, but by using a layered disk structure consisting of a bottom $\mathrm{CaF}_{2}$ layer, middle Pd layer and a top Pt cap, see Figure 3b. The addition of these extra layers has been shown to have a great impact on the long term stability of the hydrogen sensing signal. This is of vital importance if these types of sensors are to be able to compete with other types of hydrogen sensors in real applications.
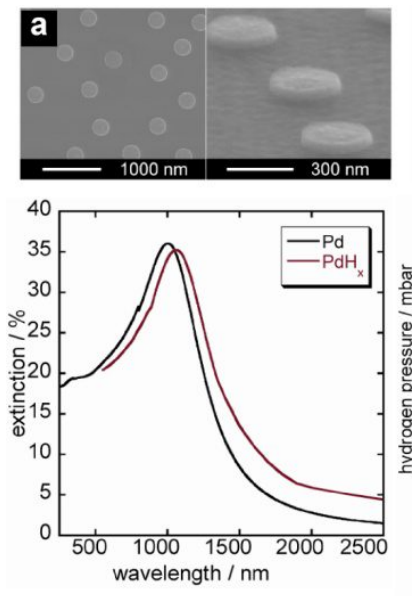
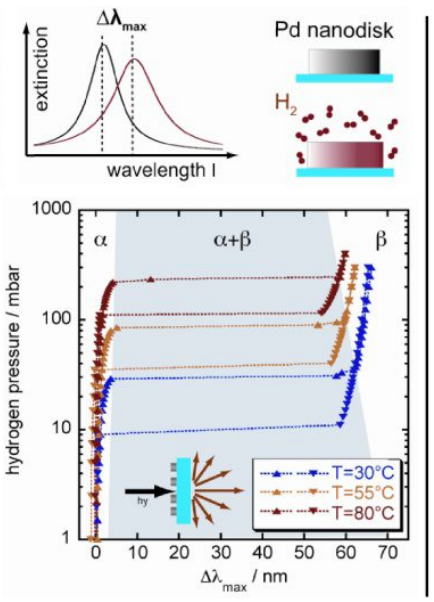
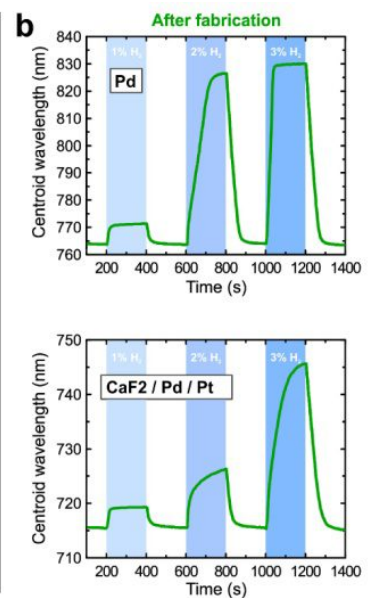
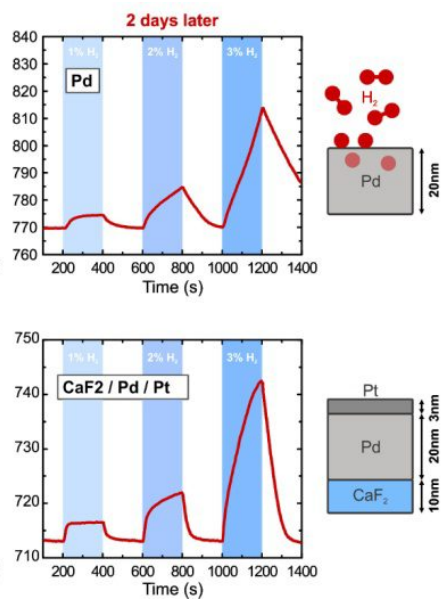

Figure 3. a) Direct sensing of hydrogen uptake and release in Pd nanodisks. By studying the shift of the LSPR upon hydrogen exposure, temperature dependent isotherm measurements can be performed. Adapted with permission from ${ }^{21}$. C 2010 WILEY-VCH Verlag GmbH \& Co. KGaA, Weinheim. b) Long time stability of a direct sensor based on hydride formation in Pd. After only two days the sensors response has deteriorated. By adding a bottom layer of $\mathrm{CaF}_{2}$ and a thin layer of Pt on top of the Pd nanodisks, the stability of the sensor is greatly enhanced. Adapted with permission from ${ }^{26}$. C) 2013 Optical Society of America.

\subsection{Indirect sensors}

In some cases the LSPR (or optical cross-section in more general terms) of the studied nanoentity is by itself too weak to be practically used for hydrogen sensing. This can arise from the fact that the material is poor when it comes to supporting LSPRs; that the particles are so small that their LSPR occurs outside of the visible spectral range; or, as can be the case for single particle studies, that they don't scatter enough light by themselves to be easily detected. In these cases it is possible to utilize other metal nanoparticles with superior and tailored LSPR properties as sensors that probe the hydride forming entities located in their vicinity via their optical near fields. In this case the LSPR nanoparticles (typically $\mathrm{Au}$ ) do not themselves interact with hydrogen, but instead act as optical antennas. We call this sensing scheme indirect sensing. 


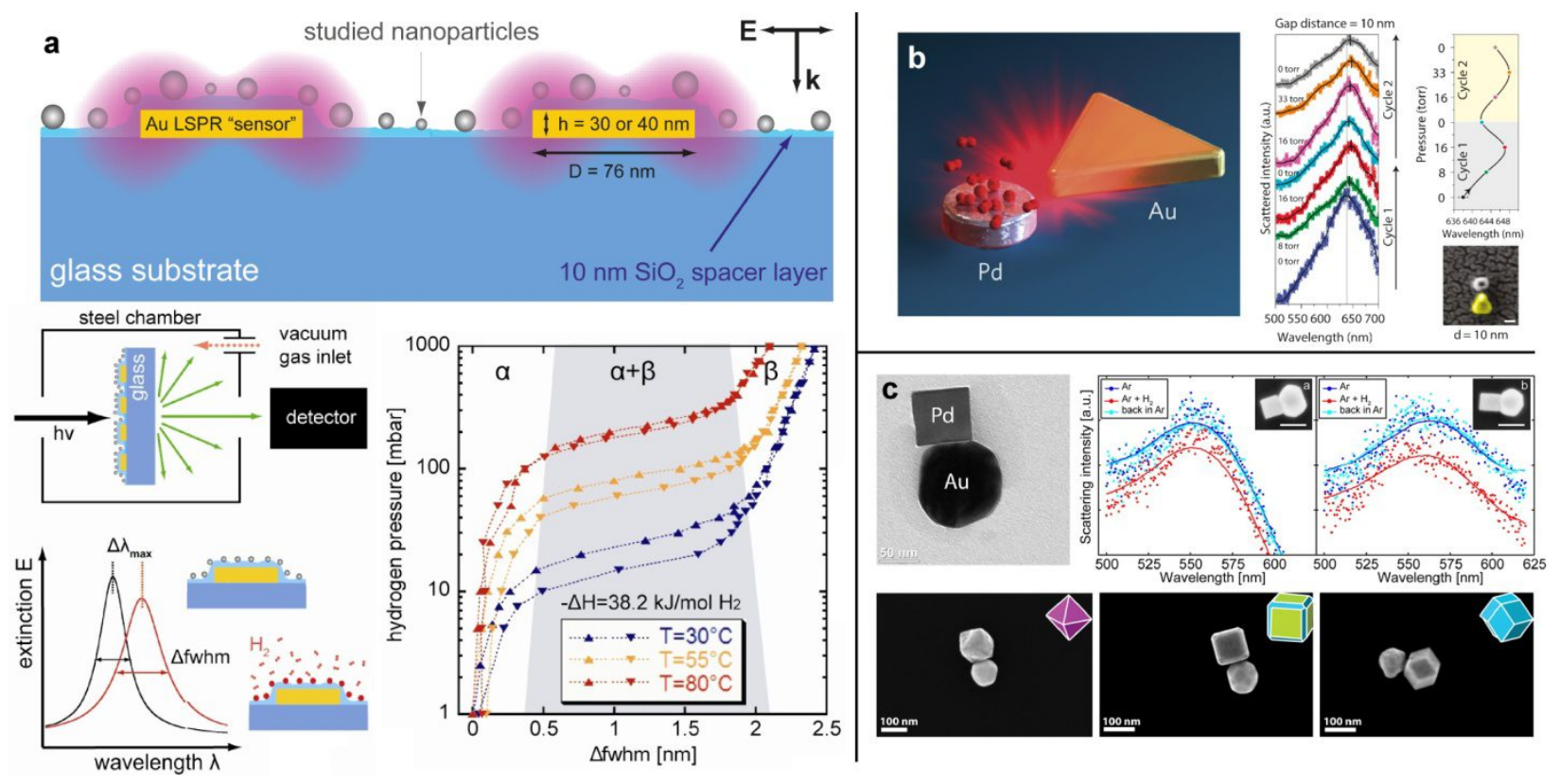

Figure 4. a) Schematic illustration of indirect nanoplasmonic sensing (INPS) used to study hydride formation and decomposition in Pd nanoparticles in the sub-10 nm size range. The plot shows optical isotherm measurements for $5 \mathrm{~nm}$ Pd nanoparticles. Adapted with permission from ${ }^{27}$. (C) 2010 American Chemical Society. b) Single particle measurements of the hydride formation and decomposition in an individual Pd disk. By fabricating a Au nanoantenna with a tailored focus next to the Pd disk it is possible to study the hydrogenation response using dark field scattering spectroscopy. Reprinted by permission from Macmillan Publishers Ltd: Nature Materials ${ }^{28}$, copyright 2011. c) Dimer nanostructures consisting of a single crystal Pd nanoparticle and a Au nanoparticle synthesized using wet chemistry and electrostatic self-assembly. The shape and surface facets of the Pd crystal can be controlled during synthesis and the attached Au nanoparticle antenna allows for single particle LSPR studies of hydrogen uptake in the adjacent Pd nanoparticle. Adapted with permission from ${ }^{29}$.

The idea to utilize indirect LSPR sensors for optical hydrogen sensing was introduced by Langhammer et al. ${ }^{27}$ after having previously been used to study catalytic reactions ${ }^{30}$. Figure 4 a shows the INPS platform used to study hydrogen uptake and release in Pd particles grown on the sensor surface. The INPS platform has since its introduction been used for detailed studies regarding the effect of nanosizing on the hydrogen interactions with Pd nanoparticles in the sub$10 \mathrm{~nm}$ size range. These studies include both the thermodynamics ${ }^{27,31,32}$ and the kinetics ${ }^{33}$ of the hydride formation and decomposition process. This has helped reach a deeper understanding of how the size of nanoparticles affects their hydrogen storage properties. Other ways to study these types of systems include volumetric methods (change in gas pressure), gravimetric methods (change in mass of the system), or x-ray diffraction (change in lattice parameter). All these other methods require relatively large sample volumes and they therefore suffer from problems related to the wide size distributions of the probed particles and related inhomogeneous sample material effects. To overcome these problems it would be beneficial if the hydrogenation measurements could be carried out on a single metal nanoparticle particle. Single particle experiments would moreover provide the possibility to characterize the studied particle in detail, making it possible to correlate any observed effects in hydrogenation behavior to particle size, shape and surface facets. It turns out that the indirect LSPR sensing concept, i.e. placing a plasmonic antenna adjacent to a hydride forming entity, makes it is possible to achieve single particle experiments. In fact, it is experimentally relatively straightforward, as only a dark field microscope is needed to illuminate and collect the scattered light from the nanoparticle of interest. However, in order for this technique to work, the sensor particle must be chosen such that it scatters light strongly enough to be detectable in the microscope. The first successful hydrogen sensing experiment on a single Pd nanoparticle was carried out by Liu et al. $^{28}$. Using e-beam lithography they fabricated triangular Au nanoantennas with a Pd nanodisk at one of its tips, in a so-called nanofocus, see Figure 4b. By tracking the LSPR scattering peak when the sample was exposed to $\mathrm{N}_{2}$ atmospheres containing various concentrations of $\mathrm{H}_{2}$ gas they were able to monitor the hydride formation in the single Pd disk. A similar approach but using a slightly different sample configuration was presented by Shegai et al. ${ }^{34}$. Here they used a stacked arrangement of $\mathrm{Au}$ nanoantennas with the hydride former on top to measure hydride formation and 
decomposition isotherms in both single Pd and single Mg nanoparticles. Both these works convincingly showed that it indeed is possible to study hydrogen uptake in single nanoentities. However, in both cases, the hydride forming Pd and $\mathrm{Mg}$ nanoparticles under scrutiny were relatively large $(30-60 \mathrm{~nm})$. As have been shown in previous studies on ensembles of particles, the interesting regime where one expects effects imposed by the particle size to become appreciable is below $10 \mathrm{~nm} .{ }^{14,35,36}$ To be able to study such small particles careful considerations have to be taken on the design of the antenna structure. One way to increase the sensitivity, as presented theoretically by Tittl et al. ${ }^{37}$ and Dasgupta et al. ${ }^{38}$, is to place the hydride forming particle in gap between two plasmonic antenna elements. In this configuration coupling of the LSPRs in the two sensor elements will give rise to region with a focused field ("hot-spot") in the gap between them. The LSPR of this coupled system will be highly sensitive to changes occurring in this hot-spot. The feasibility of this approach was recently demonstrated by Syrenova et al. ${ }^{39}$ where a antenna structure consisting of a dimer of $\mathrm{Au}$ nanodisks with a small Pd particle in the gap was used to detect hydrogen uptake in a single Pd particle with a diameter of only $16 \mathrm{~nm}$. These efforts move single particle studies closer towards the interesting sub-10 $\mathrm{nm}$ size regime.

All the previously cited experimental studies on single particles have dealt with particles fabricated using top-down nanofabrication techniques. They were chosen because they typically provide very good control of the sensor structure. However, when it comes to the structure of the hydride forming particle itself, one is somewhat limited as only particles produced using physical vapor deposition (PVD) methods are accessible. These particles are produced with only very limited control when it comes to shape and crystallinity and also size (sub $10 \mathrm{~nm}$ is very difficult). One would therefore like to be able to combine this top-down fabrication with particles synthesized in a more controlled fashion. One way to do this is to synthesize particles, which both react with hydrogen and also feature sufficiently good plasmonic properties to be able to be observed in a dark-field microscope. Tang et al. ${ }^{40}$ synthesized Au-Pd core-shell nanoparticles, which fulfill both these criteria. They were also able to control the shape of the synthesized particles and observed its effect on the hydrogen sensing properties at the single particle level. Another option than to use a hydride forming shell of a metal around a sensor particle is to attach the plasmonic sensor particle to the hydride former of interest. A first step in this direction was taken by Tittl et al. ${ }^{41}$ who synthesized $\mathrm{Au}-\mathrm{SiO}_{2}$ core-shell and dispersed them on a continuous Pd film where they acted as local plasmonic probes ${ }^{42}$. By studying the scattering from the $\mathrm{Au}-\mathrm{SiO}_{2}$ the hydrogen uptake and release in the Pd film could be monitored. Taking this idea one step further Gschneidtner et al. ${ }^{29}$ have developed a method where they were able to attach plasmonically active nanoparticles ( $\mathrm{Au}$ or $\mathrm{Ag}$ ) to shape-selected Pd nanoparticles to form dimers based on electrostatic self-assembly. Figure 4c shows examples of the achieved structures together with single particle scattering spectra response for two different particles as they are exposed to hydrogen. Using this technique Gschneidtner et al. were able to accurately control the shape and size of the Pd without having to worry about its plasmonic properties.

\subsection{Other nanoplasmonic hydrogen sensing concepts}

Apart from the measurement of a spectral shift of the characteristic LSPR peak measured in a extinction or scattering configuration, other plasmon based optical hydrogen sensors have also been presented. One example is the perfect absorber demonstrated by Tittl et al. ${ }^{43}$. In this work Pd nanowires were nanofabricated on top of a Au film covered by a $\mathrm{MgF}_{2}$ spacer layer. Given that the geometry and materials arrangements in the sample are chosen correctly it is possible to achieve almost perfect absorption of a specific wavelength of light. As hydrogen is absorbed into the Pd nanowires, the condition for the perfect absorption is no longer perfectly fulfilled and more light is reflected from the sample surface (i.e. no longer perfectly absorbed). By measuring reflection at the single wavelength where perfect absorption occurs it is thus possible to sense the presence of hydrogen. Another example of an alternative strategy is the work by Shegai et al. ${ }^{44}$ where they used a nanoplasmonic directional color routing scheme occurring in heterodimer nanostructures consisting of a $\mathrm{Au}$ and a Pd nanodisk element. The technique is based on the work presented in ${ }^{45}$, where Shegai et al. showed that nanodisk heterodimers can be designed in such a way that blue and red light is scattered in different spatial directions. This effect depends on the materials of the two nanodisk elements in the heterodimer and the phase shifts between them. Hence, in the case of the AuPd heterostructure used for hydrogen sensing, as hydrogen is absorbed into the Pd disk, the directional scattering effect changes and the presence of hydrogen is detected as a change in intensity scattered to the left and to the right. 


\section{SPR-BASED SENSORS}

The main three ways to couple light into a metal film to excite a SPR are: coupling with a prism, coupling with a grating, or using an optical waveguide ${ }^{18}$, see Figure 5a. All SPR-based hydrogen sensors work in principle in the same way (by exciting a propagating surface plasmon) and differ only in the way that the SPR is excited. The first hydrogen sensor based on SPR was demonstrated by Chadwick et al. ${ }^{46}$ in 1993 and was based on the excitation of SPRs in a Pd film using a prism. Since then several works on SPRs in Pd have been presented using various ways to excite the SPR: prism ${ }^{47,48}$, grating ${ }^{49}$ and waveguides ${ }^{50-52}$. Worth to mention is also the work by Konopsky et al. ${ }^{53}$ where they used surface waves on a photonic crystal coupled by a prism to study the hydride formation and decomposition in Pd nanoparticles in the sub$10 \mathrm{~nm}$ size range.

Another type of SPR based hydrogen sensors are based on optical fibers. The sensor is produced by simply removing the cladding of a section of the optical fiber and adding a material that will interact with hydrogen (often Pd), see Figure 5b. In this way, the light passing through the fiber will excite a SPR mode in the film, which in turn will affect the transmitted intensity of light through the fiber. This type of sensor utilizing the excitation of the SPR in a Pd film on an optical fiber was first presented by Bevenot et al. ${ }^{54}$. Since the introduction of this type of sensors, research into increasing their sensitivity has yielded several avenues, including tapering the fiber ${ }^{55}, 56$ or exchanging the Pd film for multilayer structures consisting of a bottom layer with better SPR properties (e.g. Au or Ag) and a top Pd layer separated by a dielectric spacer layer ${ }^{57-60}$ (see Figure 5 b), in resemblance to the indirect LSPR sensors.

a

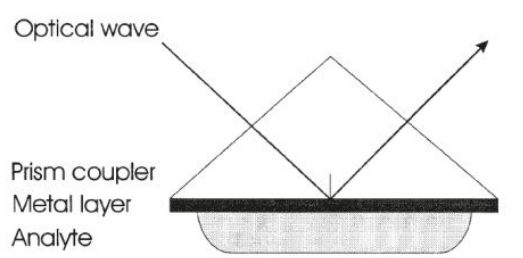

Analyte
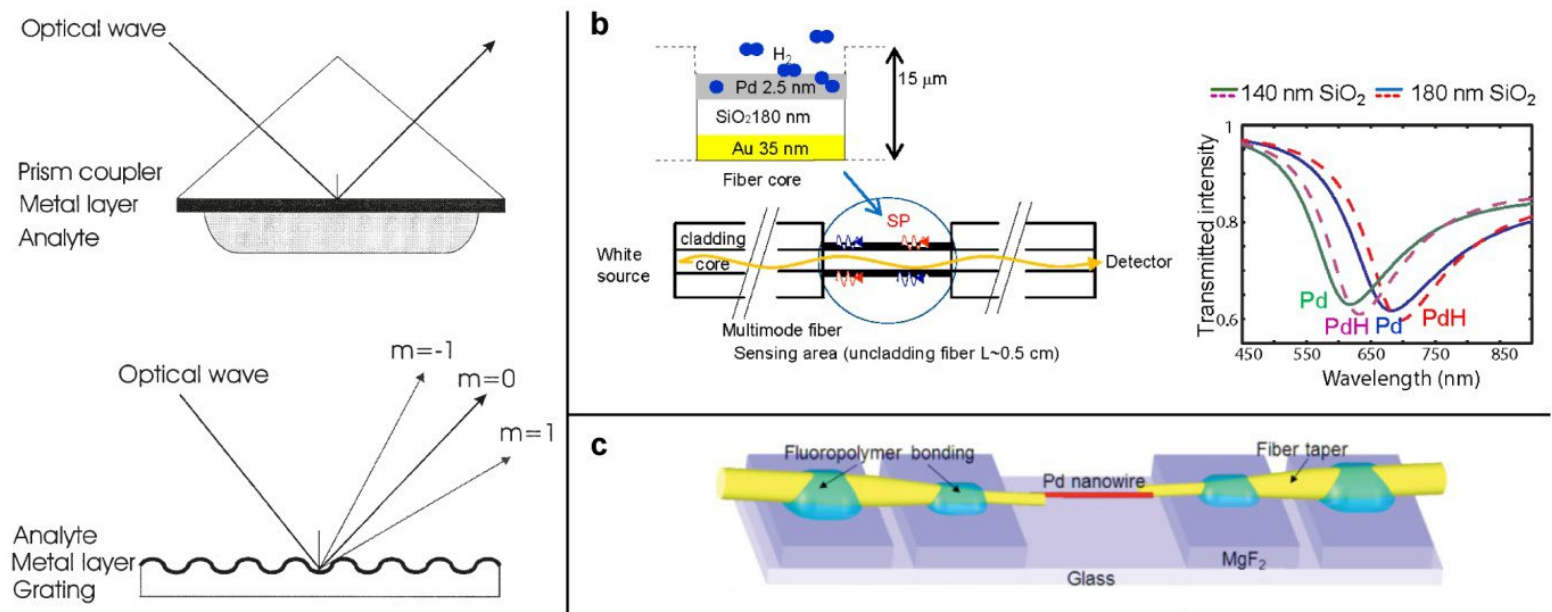

Grating
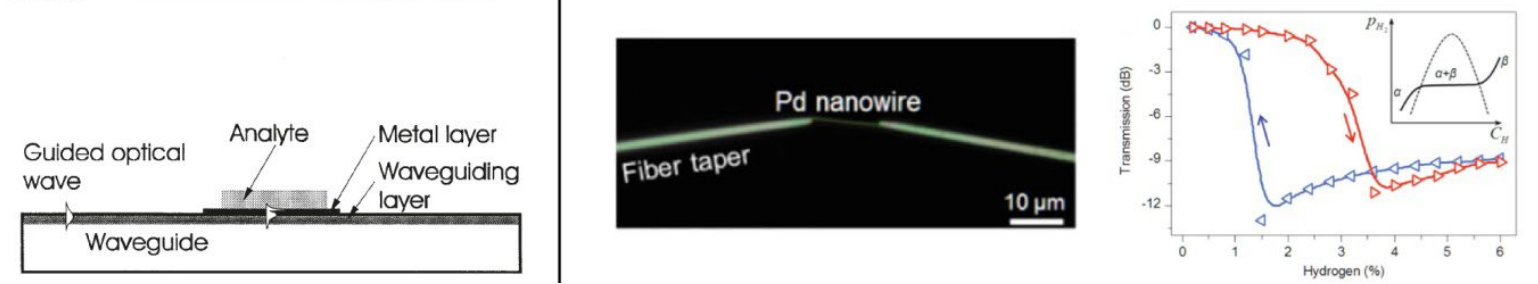

Figure 5. a) Different ways to couple light to excite a SPR mode: prism coupling, grating coupling or using a waveguide. Reprinted by permission from ${ }^{18}$. (C) 1999 Elsevier. b) Optical fiber based SPR hydrogen sensor. Part of the cladding of the fiber is replaced with an active layer and as this layer is exposed to hydrogen the intensity of transmitted light is altered. Reproduced with permission from ${ }^{60}$. (C) 2013 Optical Society of America. c) Measurement of the hydrogen uptake and release of a single Pd nanowire studied by the excitation of a SPR mode propagating along the nanowire. Adapted with permission from ${ }^{61}$. C 2013 WILEY-VCH Verlag GmbH \& Co. KGaA, Weinheim.

In the framework of SPR based hydrogen sensors, investigations to overcome some of the inherent problems with using $\mathrm{Pd}$ as the active hydrogen sensing material are ongoing. These problems arise (i) due to cross sensitivity or poisoning of the Pd sensor by other gases like, for example, carbon monoxide and (ii) due to the hysteresis exhibited during the hydride formation/decomposition, see Figure 1b. Because of this hysteresis it is not possible to get a one-to-one 
relationship between the sensor signal and the hydrogen concentration in the gas, since the response of the sensor will depend on whether the hydrogen content has been increasing or decreasing, i.e. it will depend on the history of the concentration. Both the hysteresis problem and the cross sensitivity and poisoning of the sensor need to be eliminated (or at least reduced) if a sensor is to be of practical use. One avenue that is being explored is the possibility to alloy Pd with other metals. Chadwick et al. ${ }^{62}$ investigated a SPR sensor based on a Pd/Ni alloy film to see if this alloy could better resist poisoning by for instance $\mathrm{CO}$ and $\mathrm{CH}_{4}$. Other Pd alloys for optical fiber based SPR sensors have also been investigated for their increased sensitivity at lower hydrogen concentrations such as $\mathrm{Pd} / \mathrm{Ag}^{63}$ and $\mathrm{Pd} / \mathrm{Au}$ alloys ${ }^{64,65}$. These systems have also shown smaller hysteresis for the hydride formation/decomposition ${ }^{66}$. Apart from the Pd based sensors, hydrogen sensing layers consisting of $\mathrm{ITO}^{67}$ or $\mathrm{WO}_{3} / \mathrm{Pt}$ composite films ${ }^{68}$ have also been reported.

Another interesting example of SPR based hydrogen sensing is the study of hydrogen uptake in single nanowires. Gu et al. have shown that by using two tapered optical fibers they are able to excite and read out the SPR mode propagating along a Pd coated $\mathrm{Ag}$ nanowire ${ }^{69}, \mathrm{Pd}$ nanowire, or Pd/Au alloy nanowire ${ }^{61}$, see Figure $5 \mathrm{c}$. By changing the hydrogen content in the gas around the nanowire they measure hydrogen absorption and desorption isotherms for the single nanowire, reminiscent of the single nanoparticle studies performed using dark field scattering spectroscopy.

\section{OTHER OPTICAL METHODS}

The changes in the optical properties of metals as they form hydrides are not only utilized in plasmonic hydrogen sensors, but other optical hydrogen sensors make use of this fact. In this review we will not go into detail about these techniques and works, but we would still like to highlight some of the efforts in this area. A large number of sensors have been developed based on the change in reflection or transmission of a metal film as a hydride is formed. A recent example of such a sensor structure was presented by Ngene et al. ${ }^{70}$ where they used the hydride formation in an yttrium film as the sensing element. By elegantly optimizing interference effects, with these sensors it is possible to detect the formation of the hydride by naked eye through a distinct color change of the film. Similarly, such films can also be produced on the tip of an optical fiber, which provides an easy way to introduce the sensor into the desired environment while, at the same time, carrying out a reflection measurement. These kinds of optical fiber tip sensors were first introduced by Butler ${ }^{71}$ and have since then matured greatly. As an example the work by Slaman et al. ${ }^{72,}{ }^{73}$, where magnesium based alloys were used to sense hydrogen, can be mentioned. Other optical sensors make use of the volume change of the metal upon hydride formation, for example interference based sensors. These work by either changing the optical path length for light passing through the sensor upon hydride formation ${ }^{74,75}$, or by creating a grating with the hydride forming metal and studying the change in the interference from the grating ${ }^{76-78}$. A new optical method to

measure the hydride formation in nanoparticles was presented by Bardhan et al. ${ }^{79}$. In this work they used the luminescence of the Pd nanocubes illuminated by a laser to study the hydrogen storage properties of Pd nanocrystals of different sizes.

\section{CONCLUSIONS}

Plasmonic hydrogen sensors have come a long way in the last decade and a large number of different strategies using either LSPRs or SPRs as signal transducer have been developed. From a materials science point of view when it comes to the fundamental studies and understanding of hydride formation in metal nanoparticles, in particular the LSPR based techniques have proven to be very useful and capable of studying even single nanoparticles. This is something that is hard to achieve with other techniques, especially without the need for low temperatures or low pressures. When it comes to the use of the sensors for gas-sensing applications the SPR based sensors are more mature than the LSPR ones. For the LSPR based sensors, more work when it comes to sensor stability and selectivity is needed if these are to reach commercial applications. However, work presented on other optical hydrogen sensors based on metal hydrides show that many of these problems can be overcome for instance by the use of alloys and coatings and these ideas should now be implemented in nanoplasmonic hydrogen sensors to take them to the next level. 


\section{REFERENCES}

[1] Schlapbach, L., “TECHNOLOGY Hydrogen-fuelled vehicles,” Nature, 460(7257), 809-811 (2009).

[2] Gupta, R. B., [Hydrogen fuel: production, transport and storage.] Taylor \& Francis, (2009).

[3] Wilkins, J. R., Stoner, G. E., and Boykin, E. H., "Microbial Detection Method Based on Sensing MolecularHydrogen," Applied Microbiology, 27(5), 949-952 (1974).

[4] Hitchcock, C. H. S., "Determination of hydrogen as a marker in irradiated frozen food," Journal of the Science of Food and Agriculture, 80(1), 131-136 (2000).

[5] Hurme, E. U., and Ahvenainen, R., "A nondestructive leak detection method for flexible food packages using hydrogen as a tracer gas," Journal of Food Protection, 61(9), 1165-1169 (1998).

[6] Shin, W., "Medical applications of breath hydrogen measurements," Analytical and Bioanalytical Chemistry, 19 (2014).

[7] Buttner, W. J., Post, M. B., Burgess, R., and Rivkin, C., "An overview of hydrogen safety sensors and requirements," International Journal of Hydrogen Energy, 36(3), 2462-2470 (2011).

[8] Boon-Brett, L., Bousek, J., Black, G., Moretto, P., Castello, P., Hubert, T., and Banach, U., "Identifying performance gaps in hydrogen safety sensor technology for automotive and stationary applications," International Journal of Hydrogen Energy, 35(1), 373-384 (2010).

[9] Hubert, T., Boon-Brett, L., Black, G., and Banach, U., "Hydrogen sensors - A review," Sensors and Actuators B-Chemical, 157(2), 329-352 (2011).

[10] Ando, M., "Recent advances in optochemical sensors for the detection of $\mathrm{H}-2, \mathrm{O}-2, \mathrm{O}-3, \mathrm{CO}, \mathrm{CO} 2$ and $\mathrm{H} 2 \mathrm{O}$ in air," Trac-Trends in Analytical Chemistry, 25(10), 937-948 (2006).

[11] Fukai, Y., [The metal-hydrogen system] Springer-Verlag, (2005).

[12] Schlapbach, L., and Zuttel, A., "Hydrogen-storage materials for mobile applications," Nature, 414(6861), 353$358(2001)$.

[13] Oumellal, Y., Rougier, A., Nazri, G. A., Tarascon, J. M., and Aymard, L., "Metal hydrides for lithium-ion batteries," Nature Materials, 7(11), 916-921 (2008).

[14] Berube, V., Radtke, G., Dresselhaus, M., and Chen, G., "Size effects on the hydrogen storage properties of nanostructured metal hydrides: A review," International Journal of Energy Research, 31(6-7), 637-663 (2007).

[15] Schwarz, R. B., and Khachaturyan, A. G., "Thermodynamics of open two-phase systems with coherent interfaces: Application to metal-hydrogen systems," Acta Materialia, 54(2), 313-323 (2006).

[16] Faraday, M., "The bakerian lecture: Experimental relations of gold (and other metals) to light.," Phil. Trans. R. Soc. Lond., 147, 145-181 (1857).

[17] Kelly, K. L., Coronado, E., Zhao, L. L., and Schatz, G. C., "The optical properties of metal nanoparticles: The influence of size, shape, and dielectric environment," Journal of Physical Chemistry B, 107(3), 668-677 (2003).

[18] Homola, J., Yee, S. S., and Gauglitz, G., "Surface plasmon resonance sensors: review," Sensors and Actuators B-Chemical, 54(1-2), 3-15 (1999).

[19] Silkin, V. M., Muino, R. D., Chernov, I. P., Chulkov, E. V., and Echenique, P. M., "Tuning the plasmon energy of palladium-hydrogen systems by varying the hydrogen concentration," Journal of Physics-Condensed Matter, 24(10), (2012).

[20] Langhammer, C., Zoric, I., and Kasemo, B., "Hydrogen storage in Pd nanodisks characterized with a novel nanoplasmonic sensing scheme," Nano Letters, 7(10), 3122-3127 (2007).

[21] Zoric, I., Larsson, E. M., Kasemo, B., and Langhammer, C., "Localized Surface Plasmons Shed Light on Nanoscale Metal Hydrides," Advanced Materials, 22(41), 4628-4633 (2010).

[22] Larsson, E. M., Edvardsson, M. E. M., Langhammer, C., Zoric, I., and Kasemo, B., "A combined nanoplasmonic and electrodeless quartz crystal microbalance setup," Review of Scientific Instruments, 80(12), (2009).

[23] Poyli, M. A., Silkin, V. M., Chernov, I. P., Echenique, P. M., Muino, R. D., and Aizpurua, J., "Multiscale Theoretical Modeling of Plasmonic Sensing of Hydrogen Uptake in Palladium Nanodisks," Journal of Physical Chemistry Letters, 3(18), 2556-2561 (2012).

[24] Langhammer, C., Larsson, E. M., Zhdanov, V. P., and Zoric, I., "Asymmetric Hysteresis in Nanoscopic SingleMetal Hydrides: Palladium Nanorings," Journal of Physical Chemistry C, 116(40), 21201-21207 (2012).

[25] Niu, W. X., Zhang, W. Q., Firdoz, S., and Lu, X. M., "Controlled Synthesis of Palladium Concave Nanocubes with Sub-10-Nanometer Edges and Corners for Tunable Plasmonic Property," Chemistry of Materials, 26(6), 2180-2186 (2014). 
[26] Strohfeldt, N., Tittl, A., and Giessen, H., "Long-term stability of capped and buffered palladium-nickel thin films and nanostructures for plasmonic hydrogen sensing applications," Optical Materials Express, 3(2), 194204 (2013).

[27] Langhammer, C., Larsson, E. M., Kasemo, B., and Zoric, I., "Indirect Nanoplasmonic Sensing: Ultrasensitive Experimental Platform for Nanomaterials Science and Optical Nanocalorimetry," Nano Letters, 10(9), 3529$3538(2010)$.

[28] Liu, N., Tang, M. L., Hentschel, M., Giessen, H., and Alivisatos, A. P., "Nanoantenna-enhanced gas sensing in a single tailored nanofocus," Nature Materials, 10(8), 631-636 (2011).

[29] Gschneidtner, T. A., Fernandez, Y. A. D., Syrenova, S., Westerlund, F., Langhammer, C., and Moth-Poulsen, K., "A versatile self-assembly strategy for the synthesis of shape-selected colloidal noble metal nanoparticle heterodimers," Langmuir, 30(11), 3041-3050 (2014).

[30] Larsson, E. M., Langhammer, C., Zoric, I., and Kasemo, B., "Nanoplasmonic Probes of Catalytic Reactions," Science, 326(5956), 1091-1094 (2009).

[31] Langhammer, C., Zhdanov, V. P., Zoric, I., and Kasemo, B., "Size-dependent hysteresis in the formation and decomposition of hydride in metal nanoparticles," Chemical Physics Letters, 488(1-3), 62-66 (2010).

[32] Wadell, C., Pingel, T., Olsson, E., Zorić, I., Zhdanov, V. P., and Langhammer, C., "Thermodynamics of hydride formation and decomposition in supported sub-10 nm Pd nanoparticles of different sizes," Chemical Physics Letters, 603, 75-81 (2014).

[33] Langhammer, C., Zhdanov, V. P., Zoric, I., and Kasemo, B., "Size-Dependent Kinetics of Hydriding and Dehydriding of Pd Nanoparticles," Physical Review Letters, 104(13), (2010).

[34] Shegai, T., and Langhammer, C., "Hydride Formation in Single Palladium and Magnesium Nanoparticles Studied By Nanoplasmonic Dark-Field Scattering Spectroscopy,” Advanced Materials, 23(38), 4409-+ (2011).

[35] Pundt, A., Sachs, C., Winter, M., Reetz, M. T., Fritsch, D., and Kirchheim, R., "Hydrogen sorption in elastically soft stabilized Pd-clusters," Journal of Alloys and Compounds, 293, 480-483 (1999).

[36] Zuttel, A., Nutzenadel, C., Schmid, G., Chartouni, D., and Schlapbach, L., "Pd-cluster size effects of the hydrogen sorption properties," Journal of Alloys and Compounds, 293, 472-475 (1999).

[37] Tittl, A., Kremers, C., Dorfmuller, J., Chigrin, D. N., and Giessen, H., "Spectral shifts in optical nanoantennaenhanced hydrogen sensors," Optical Materials Express, 2(2), 111-118 (2012).

[38] Dasgupta, A., and Kumar, G. V. P., "Palladium bridged gold nanocylinder dimer: plasmonic properties and hydrogen sensitivity," Applied Optics, 51(11), 1688-1693 (2012).

[39] Syrenova, S., Wadell, C., and Langhammer, C., "Shrinking-hole colloidal lithography: Self-aligned nanofabrication of complex plasmonic nanoantennas," Nano Letters, 14(5), 2655-2663 (2014).

[40] Tang, M. L., Liu, N., Dionne, J. A., and Alivisatos, A. P., "Observations of Shape-Dependent Hydrogen Uptake Trajectories from Single Nanocrystals," Journal of the American Chemical Society, 133(34), 13220-13223 (2011).

[41] Tittl, A., Yin, X. H., Giessen, H., Tian, X. D., Tian, Z. Q., Kremers, C., Chigrin, D. N., and Liu, N., "Plasmonic Smart Dust for Probing Local Chemical Reactions,” Nano Letters, 13(4), 1816-1821 (2013).

[42] Li, J. F., Huang, Y. F., Ding, Y., Yang, Z. L., Li, S. B., Zhou, X. S., Fan, F. R., Zhang, W., Zhou, Z. Y., Wu, D. Y., Ren, B., Wang, Z. L., and Tian, Z. Q., "Shell-isolated nanoparticle-enhanced Raman spectroscopy," Nature, 464(7287), 392-395 (2010).

[43] Tittl, A., Mai, P., Taubert, R., Dregely, D., Liu, N., and Giessen, H., "Palladium-Based Plasmonic Perfect Absorber in the Visible Wavelength Range and Its Application to Hydrogen Sensing," Nano Letters, 11(10), 4366-4369 (2011).

[44] Shegai, T., Johansson, P., Langhammer, C., and Kall, M., "Directional Scattering and Hydrogen Sensing by Bimetallic Pd-Au Nanoantennas," Nano Letters, 12(5), 2464-2469 (2012).

[45] Shegai, T., Chen, S., Miljkovic, V. D., Zengin, G., Johansson, P., and Kall, M., “A bimetallic nanoantenna for directional colour routing," Nature Communications, 2, (2011).

[46] Chadwick, B., and Gal, M., "Enhanced Optical-Detection of Hydrogen Using the Excitation of SurfacePlasmons in Palladium," Applied Surface Science, 68(1), 135-138 (1993).

[47] Konopsky, V. N., Basmanov, D. V., Alieva, E. V., Dolgy, D. I., Olshansky, E. D., Sekatskii, S. K., and Dietler, G., "Registration of long-range surface plasmon resonance by angle-scanning feedback and its implementation for optical hydrogen sensing," New Journal of Physics, 11, (2009).

[48] Morjan, M., Zuchner, H., and Cammann, K., "Contributions to a reliable hydrogen sensor based on surface plasmon surface resonance spectroscopy,” Surface Science, 603(10-12), 1353-1359 (2009). 
[49] Lin, K. Q., Lu, Y. H., Chen, J. X., Zheng, R. S., Wang, P., and Ming, H., "Surface plasmon resonance hydrogen sensor based on metallic grating with high sensitivity," Optics Express, 16(23), 18599-18604 (2008).

[50] Tobiska, P., Hugon, O., Trouillet, A., and Gagnaire, H., "An integrated optic hydrogen sensor based on SPR on palladium," Sensors and Actuators B-Chemical, 74(1-3), 168-172 (2001).

[51] Fong, N. R., Berini, P., and Tait, R. N., "Modeling and design of hydrogen gas sensors based on a membranesupported surface plasmon waveguide," Sensors and Actuators B-Chemical, 161(1), 285-291 (2012).

[52] Alam, M. Z., Carriere, N., Bahrami, F., Mojahedi, M., and Aitchison, J. S., "Pd-based integrated optical hydrogen sensor on a silicon-on-insulator platform," Optics Letters, 38(9), 1428-1430 (2013).

[53] Konopsky, V. N., Basmanov, D. V., Alieva, E. V., Sekatskii, S. K., and Dietler, G., "Size-dependent hydrogen uptake behavior of Pd nanoparticles revealed by photonic crystal surface waves," Applied Physics Letters, 100(8), (2012).

[54] Bevenot, X., Trouillet, A., Veillas, C., Gagnaire, H., and Clement, M., "Surface plasmon resonance hydrogen sensor using an optical fibre," Measurement Science \& Technology, 13(1), 118-124 (2002).

[55] Villatoro, J., Luna-Moreno, D., and Monzon-Hernandez, D., "Optical fiber hydrogen sensor for concentrations below the lower explosive limit," Sensors and Actuators B-Chemical, 110(1), 23-27 (2005).

[56] Villatoro, J., and Monzon-Hernandez, D., "Fast detection of hydrogen with nano fiber tapers coated with ultra thin palladium layers," Optics Express, 13(13), 5087-5092 (2005).

[57] Perrotton, C., Javahiraly, N., Slaman, M., Dam, B., and Meyrueis, P., "Fiber optic Surface Plasmon Resonance sensor based on wavelength modulation for hydrogen sensing," Optics Express, 19(S6), A1175-A1183 (2011).

[58] Bhatia, P., and Gupta, B. D., "Surface plasmon resonance based fiber optic hydrogen sensor utilizing wavelength interrogation," Third Asia Pacific Optical Sensors Conference, 8351, (2012).

[59] Hosoki, A., Nishiyama, M., Igawa, H., Seki, A., Choi, Y., and Watanabe, K., "A surface plasmon resonance hydrogen sensor using $\mathrm{Au} / \mathrm{Ta} 2 \mathrm{O} 5 / \mathrm{Pd}$ multi-layers on hetero-core optical fiber structures," Sensors and Actuators B, 185, 53-58 (2013).

[60] Perrotton, C., Westerwaal, R. J., Javahiraly, N., Slaman, M., Schreuders, H., Dam, B., and Meyrueis, P., “A reliable, sensitive and fast optical fiber hydrogen sensor based on surface plasmon resonance," Optics Express, 21(1), 382-390 (2013).

[61] Gu, F., Zeng, H., Zhu, Y. B., Yang, Q., Ang, L. K., and Zhuang, S., "Single-crystal Pd and its alloy nanowires for plasmon propagation and highly sensitive hydrogen detection," Advanced Optical Materials, 2(2), 189-196 (2014).

[62] Chadwick, B., Tann, J., Brungs, M., and Gal, M., "A Hydrogen Sensor-Based on the Optical-Generation of Surface-Plasmons in a Palladium Alloy," Sensors and Actuators B-Chemical, 17(3), 215-220 (1994).

[63] Hu, J. D., Jiang, M., and Lin, Z. L., "Novel technology for depositing a Pd-Ag alloy film on a tapered optical fibre for hydrogen sensing," Journal of Optics a-Pure and Applied Optics, 7(10), 593-598 (2005).

[64] Luna-Moreno, D., and Monzon-Hernandez, D., "Effect of the Pd-Au thin film thickness uniformity on the performance of an optical fiber hydrogen sensor," Applied Surface Science, 253(21), 8615-8619 (2007).

[65] Luna-Moreno, D., Monzon-Hernandez, D., Villatoro, J., and Badenes, G., "Optical fiber hydrogen sensor based on core diameter mismatch and annealed Pd-Au thin films," Sensors and Actuators B-Chemical, 125(1), 66-71 (2007).

[66] Westerwaal, R. J., Rooijmans, J. S. A., Leclercq, L., Gheorghe, D. G., Radeva, T., Mooij, L., Mak, T., Polak, L., Slaman, M., Dam, B., and Rasing, T., "Nanostructured Pd-Au based fiber optic sensors for probing hydrogen concentrations in gas mixtures," International Journal of Hydrogen Energy, 38(10), 4201-4212 (2013).

[67] Mishra, S. K., and Gupta, B. D., "Surface Plasmon Resonance-Based Fiber-Optic Hydrogen Gas Sensor Utilizing Indium-Tin Oxide (ITO) Thin Films," Plasmonics, 7(4), 627-632 (2012).

[68] Wang, X. G., Tang, Y. K., Zhou, C. D., and Liao, B., "Design and optimization of the optical fiber surface plasmon resonance hydrogen sensor based on wavelength modulation," Optics Communications, 298, 88-94 (2013).

[69] Gu, F. X., Zeng, H. P., Tong, L. M., and Zhuang, S. L., "Metal single-nanowire plasmonic sensors," Optics Letters, 38(11), 1826-1828 (2013).

[70] Ngene, P., Radeva, T., Slaman, M., Westerwaal, R. J., Schreuders, H., and Dam, B., "Seeing Hydrogen in Colors: Low-Cost and Highly Sensitive Eye Readable Hydrogen Detectors," Advanced Functional Materials, (2013). 
[71] Butler, M. A., "Fiber Optic Sensor for Hydrogen Concentrations near the Explosive Limit," Journal of the Electrochemical Society, 138(9), L46-L47 (1991).

[72] Slaman, M., Dam, B., Pasturel, M., Borsa, D. M., Schreuders, H., Rector, J. H., and Griessen, R., "Fiber optic hydrogen detectors containing Mg-based metal hydrides," Sensors and Actuators B-Chemical, 123(1), 538-545 (2007).

[73] Slaman, M., Dam, B., Schreuders, H., and Griessen, R., "Optimization of Mg-based fiber optic hydrogen detectors by alloying the catalyst," International Journal of Hydrogen Energy, 33(3), 1084-1089 (2008).

[74] Butler, M. A., "Optical Fiber Hydrogen Sensor," Applied Physics Letters, 45(10), 1007-1009 (1984).

[75] Yang, Z., Zhang, M., Liao, Y. B., Tian, Q., Li, Q. S., Zhang, Y., and Zhuang, Z., "Extrinsic Fabry-Perot interferometric optical fiber hydrogen detection system," Applied Optics, 49(15), 2736-2740 (2010).

[76] Trouillet, A., Marin, E., and Veillas, C., "Fibre gratings for hydrogen sensing," Measurement Science \& Technology, 17(5), 1124-1128 (2006).

[77] Gupta, R., Sagade, A. A., and Kulkarni, G. U., "A low cost optical hydrogen sensing device using nanocrystalline Pd grating," International Journal of Hydrogen Energy, 37(11), 9443-9449 (2012).

[78] Dai, J. X., Yang, M. H., Yu, X., and Lu, H., "Optical hydrogen sensor based on etched fiber Bragg grating sputtered with Pd/Ag composite film," Optical Fiber Technology, 19(1), 26-30 (2013).

[79] Bardhan, R., Hedges, L. O., Pint, C. L., Javey, A., Whitelam, S., and Urban, J. J., "Uncovering the intrinsic size dependence of hydriding phase transformations in nanocrystals," Nature Materials, 12(10), 905-912 (2013). 